\title{
The expression status and prognostic significance of programmed cell death I ligand I in gastro- intestinal tract cancer: a systematic review and meta-analysis
}

\author{
This article was published in the following Dove Press journal: \\ OncoTargets and Therapy \\ 15 September 2015 \\ Number of times this article has been viewed
}

\section{Baohua Huang* \\ Lei Chen* \\ Cuixia Bao \\ Chengming Sun \\ Jie $\mathrm{Li}$ \\ Lipeng Wang \\ Xia Zhang}

Department of Laboratory Medicine. Yantai Yuhuangding Hospital, Yantai,

People's Republic of China

*These authors contributed equally to this work
Correspondence: Xia Zhang Department of Laboratory Medicine, Yantai Yuhuangding Hospital, No. 20, Shudong Road, Yantai City, 264000, People's Republic of China

Email xiazhangzx@sina.com
Background: Programmed cell death 1 ligand 1 (PD-L1) expression has been observed in various malignancies. However, the association between PD-L1 expression and the survival of patients with gastrointestinal tract cancer remains controversial. Besides, the rate of PD-L1 positivity on tumor cells of digestive tract cancer is not clear. Thus, we performed a meta-analysis by incorporating all available evidence to evaluate the rate of PD-L1 positivity and the overall survival (OS) according to PD-L1 status in patients with gastrointestinal tract cancer.

Methods: Electronic databases were searched for eligible literature. Hazard ratios (HRs) for OS with 95\% confidence intervals (CIs) according to the expression status of PD-L1 evaluated by immunohistochemistry were extracted. The outcomes were synthesized based on a random-effects model.

Results: Fifteen studies (only nine reported OS) that involved 2,993 gastrointestinal tract cancer patients stratified by PD-L1 status were eligible for inclusion in our study. We found the PD-L1positive expression rate was 0.495 (95\% CI $0.415-0.576)$ if $10 \%$ was taken as the cut-off value. When the H-score method was used to evaluate PD-L1 expression, it showed that the PD-L1 positive rate was $0.639(95 \% \mathrm{CI} 0.490-0.765)$ if the cut-off value was $<50$, which was higher than when using $>50$ as the cut-off point $(0.449,95 \%$ CI $0.417-0.483)$. Additionally, PD-L1-positive gastrointestinal tract cancer patients were associated with significantly poorer OS when compared to negative ones (HR 1.61, 95\% CI 1.10-2.35, $P=0.014$ ). Subgroup analysis presented similar significant results in patients with esophageal cancer (HR 2.56, 95\% CI 1.55-4.21, $P<0.001$ ).

Conclusion: The positive expression rate of PD-L1 was nearly $50 \%$ no matter which method for immunohistochemistry evaluation we chose. Additionally, positive PD-L1 expression status in tumor cells is a risk factor for prognosis of gastrointestinal tract cancer, especially esophageal cancer.

Keywords: prognosis, esophageal cancer, immunohistochemistry, PD-L1-positive expression rate

\section{Introduction}

Gastrointestinal tract cancer refers to malignant conditions of the gastrointestinal tract, including of the esophagus, stomach, small intestine, large intestine, rectum, and anus. Among those cancer patients, colorectal carcinoma, gastric cancer, and esophageal cancer are common types (the third, fourth, and sixth most-frequently diagnosed malignancies, respectively) globally. ${ }^{1-3}$ Although recent advances in multidisciplinary therapies have improved treatment outcomes, the overall prognosis for gastrointestinal tract cancer remains poor. It is well known that the development 
and prognosis of malignant tumors are closely related to host immune functions. ${ }^{4}$ Thus, novel therapeutic strategies, especially immunotherapy, are needed to be developed and established. ${ }^{4}$

It has been identified that immune escape plays an important role in tumor progression. ${ }^{5}$ Improved understanding of the molecular mechanisms that govern the host response to tumors has led to the identification of checkpoint signaling pathways that limit the anticancer immune response. ${ }^{6}$ A particularly important immune checkpoint that mediates tumor-induced immune suppression is the binding of programmed death 1 (PD-1) expressed on tumor-infiltrating lymphocytes and its ligand 1 (PD-L1) expressed on tumor cells. ${ }^{7}$ PD-L1 has been reported to inhibit the proliferation of activated T-cells and induce the apoptosis of T-cells to form and maintain an immunosuppressive microenvironment since PD-L1 can recognize and bind the PD-1 on tumor-infiltrating lymphocytes. ${ }^{8}$

PD-L1 expression has been observed in various malignancies. Moreover, several meta-analyses have proved that PD-L1 overexpression indicates a poor prognosis for patients with non-small cell lung cancer. ${ }^{9-11}$ However, the association between PD-L1 expression and the survival of patients with gastrointestinal tract cancer remains controversial. Besides, the rate of PD-L1 positivity on tumor cells of digestive tract cancer is not clear. Therefore, the expression status and prognostic significance of PD-L1 require further comprehensive study to clarify. Thus, we performed a meta-analysis by incorporating all available evidence to evaluate the expression rate of PD-L1 and the overall survival (OS) according to PD-L1 status in patients with gastrointestinal tract cancer.

\section{Materials and methods Literature search}

Our institutions Ethics Committee has exempted our study from Institutional Review Board approval as our study involves exclusively preexisting anonymous data. The preferred reporting items for systematic reviews and metaanalyses (PRISMA) statement for reporting systematic reviews recommended by the Cochrane Collaboration was followed for conducting this meta-analysis. Two authors independently carried out a comprehensive systematic search for published articles or abstracts by searching through PubMed, Embase, Scopus, and the Cochrane Library from inception to April 2015. Searches were limited to human studies, using a combination of the terms "PD-L1", “CD274", "B7-H1", "programmed cell death 1 ligand 1", "gastrointestinal", "esophageal”, "gastric", "colorectal", "outcome", "survival”, and "prognosis". We also manually reviewed relevant reference lists and reviews. There were no language restrictions.

\section{Inclusion and exclusion criteria}

Studies meeting the following criteria were eligible for the single-arm meta-analysis of PD-L1-positive expression rate on tumor cells: (1) cohort studies which investigated PD-L1 expression level in gastrointestinal tract cancer patients; (2) the expression level of PD-L1 was tested by immunohistochemistry (IHC) staining in the tissue specimens; (3) samples of PD-L1 positive and PD-L1 negative were available. Moreover, studies in the meta-analysis that focused on patients' survival according to PD-L1 status met the following criteria: (1) studies meeting the criteria of meta-analysis for PD-L1-positive expression rate; (2) survival data stratified by PD-L1 status was available. Studies that failed to meet the inclusion criteria were excluded.

\section{Outcome measures, data extraction, and quality assessment}

The primary outcome for this meta-analysis was PD-L1-positive expression rate and OS. OS data were extracted in the form of hazard ratios (HRs) with the corresponding 95\% confidence interval (CI). If the HR was not displayed directly, it was estimated according to the methods described by a previously published article. $^{12}$ The data collection and assessment of methodological quality followed the quality of reporting of meta-analyses (QUORUM) and the Cochrane Collaboration guidelines (http:// www.cochrane.de). The following main items were abstracted from the included studies: author, year, tumor type, IHC evaluation method and cut-off value, sample size of total patients and PD-L1-positive group, and survival data. Two reviewers used the modified Newcastle-Ottawa scale to assess these studies. ${ }^{13}$ All investigators discussed and resolved all discrepancies in the extracted data. All of the eligible studies were of high quality.

\section{Statistical analysis}

All calculations for PD-L1-positive expression rate on tumor cells were performed using Meta-Analyst Beta (v 3.13; Tufts Medical Center, Boston, MA, USA). HRs for OS with 95\% CIs according to the expression status of PD-L1 was pooled using STATA (v 11.0; StataCorp, College Station, TX, USA). Heterogeneity across the incorporated studies was assessed with a forest plot and the inconsistency statistic $\left(I^{2}\right)$. A random-effects model was employed in case of potential heterogeneity and to avoid underestimation of standard errors of pooled estimates in this meta-analysis. Subgroup analysis was conducted according to IHC evaluation method and cut-off value and tumor type. An HR that was greater than 1 reflected shorter OS for PD-L1-positive patients. All CIs had two-sided probability coverage of $95 \%$. A statistical test with a $P$-value less than 0.05 was considered as significant. 


\section{Publication bias}

An extensive search strategy was made to minimize the potential publication bias. Graphical funnel plots were generated to visually assess publication bias. The statistical method to detect funnel plot asymmetry was Begg's test. ${ }^{14}$

\section{Results}

\section{Eligible studies}

In total, 574 records were identified according to the search strategy. Finally, we enrolled 15 studies $^{15-29}$ that involved 2,993 gastrointestinal tract cancer patients $(1,461 / 1,532$ cases for PD-L1 positive/negative) with available PD-L1 expression data stratified by PD-L1 status. Nine of the studies ${ }^{16-19,21,23,26-28}$ reported HRs for OS as a clinical outcome. Figure 1 summarizes the flow chart of study selection. Our study covered three major types of gastrointestinal tract cancer, esophageal cancer, gastric cancer, and colorectal carcinoma. The H-score was one of the most common methods used to evaluate the expression of PD-L1 in the tumor cells among the included articles, as well as the percentage of positively stained cells. The characteristics of the enrolled studies are shown in Table 1.

\section{Single-arm meta-analysis of positive expression rate of PD-LI}

After stratifying the enrolled studies according to IHC evaluation method and cut-off value, we found the PD-L1-positive expression rate was 0.495 (95\% CI $0.415-0.576)$ if $10 \%$ was taken as the cut-off value. When the H-score method was used to evaluate PD-L1 expression, it showed that the PD-L1 positive rate was 0.639 (95\% CI $0.490-0.765)$ if the cut-off value was $<50$, which was higher than when $>50$ was used as the cut-off point $(0.449,95 \%$ CI $0.417-0.483)$ (Figure 2).

\section{Meta-analysis of $\mathrm{PD}$-LI positive versus PD-LI negative in terms of OS}

Overall, positive expression of PD-L1 in the tumor cells of gastrointestinal tract cancer patients was associated with a significantly poorer OS than negative expression (HR 1.61, 95\% CI 1.10-2.35, $P=0.014$ ) (Figure 3).

\section{Subgroup analyses, sensitivity analyses, and publication bias}

The H-score evaluation method showed inferior survival in the PD-L1-positive group if we took $<50$ as the cutoff value (HR 1.75, 95\% CI 1.33-2.31, $P<0.001$ ). The results were similar when using $>50$ as the cut-off value (HR 1.79, 95\% CI 0.69-4.62, $P=0.231$ ). However, an only slightly numerical adverse prognostic effect of PD-L1positive expression was presented if $10 \%$ was taken as the cut-off value using the percentage method (HR 1.05, 95\% CI 0.42-2.61, $P=0.919$ ) (Figure 4). Additionally, when stratifying studies according to tumor type, we observed that meta-analysis presented significant poorer OS in the PD-L1-positive group of patients with esophageal cancer (HR 2.56, 95\% CI 1.55-4.21, $P<0.001$ ). Similar numerical survival benefits were found in the PD-L1 negative

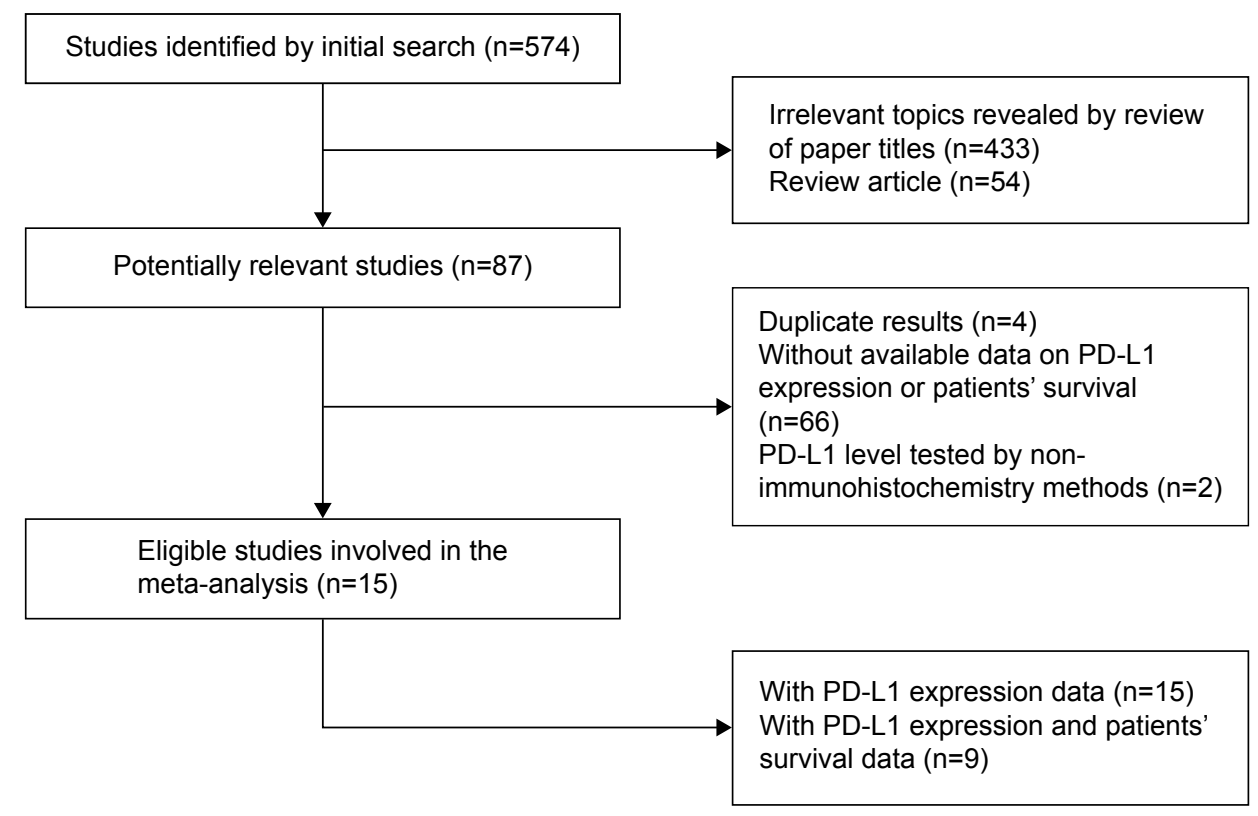

Figure I Flow chart of study selection.

Abbreviation: PD-LI, programmed cell death I ligand I. 


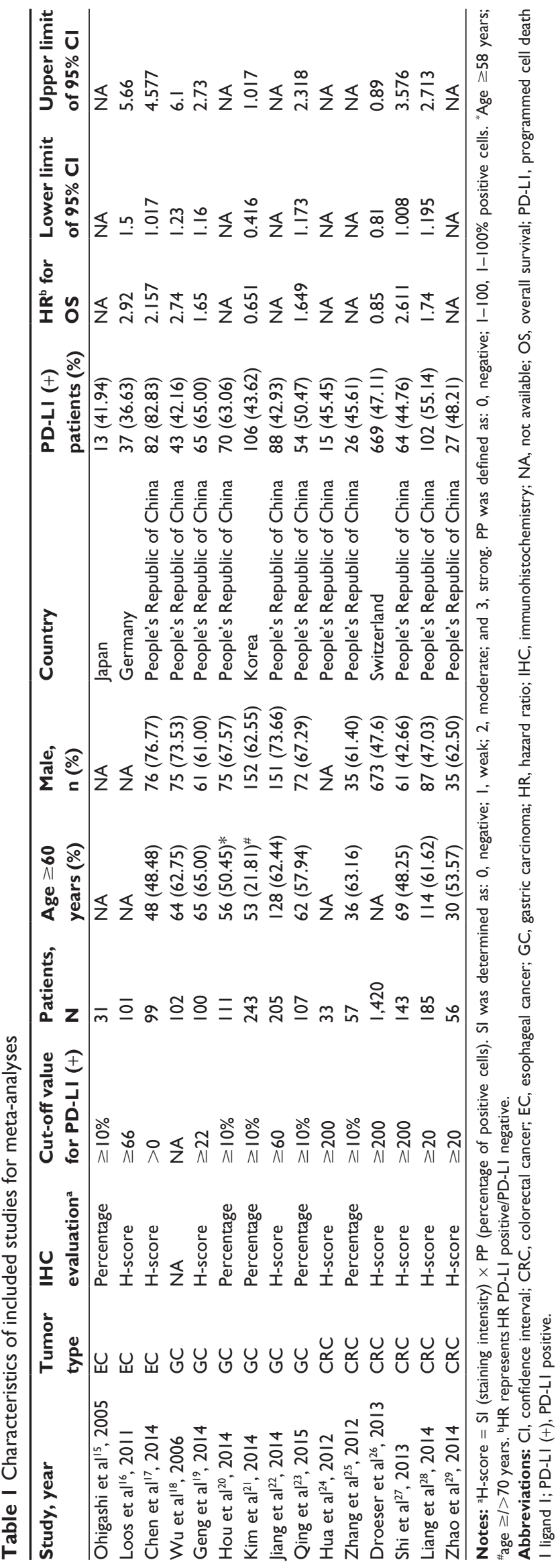

groups of patients with gastric cancer (HR 1.42, 95\% CI 0.84-2.41, $P=0.194$ ) and colorectal carcinoma (HR 1.49, 95\% CI 0.74-3.00, $P=0.260$ ) (Figure 4). Funnel plots with Begg's test are shown in Figure S1.

\section{Discussion}

For patients with gastrointestinal tract malignancies, the association of the expression of PD-L1 and their prognosis remains unclear. In addition, the positive expression rate of PD-L1 in the tumor cells of digestive tract cancer is still unknown. A meta-analysis incorporating all available data from correlative studies is a reasonable method by which to address these questions. We conducted this study and found nearly $50 \%$ of gastrointestinal tract cancer patients showed positive expression of PD-L1. Furthermore, patients with positive expression of PD-L1 had significantly poorer survival than with those with negative expression.

It is notable that the PD-L1 expression rate was delineated less when stricter criteria for positive PD-L1 expression were applied. We could see that studies using a cut-off value $>50$ for H-score ${ }^{16,22,24,26,27}$ showed a lower positive PD-L1 expression rate than those using $<50 .{ }^{17,19,28,29} \mathrm{~A}$ similar result was found if $10 \%$ was taken as the cut-off value when using the percentage method. Since IHC evaluation methods and cut-off values were not consistent for each gastrointestinal tract cancer type, subgroup meta-analysis was not possible when studies were stratified by tumor type. We could also see that studies using a cut-off value $>50$ for $\mathrm{H}$-score ${ }^{16,26,27}$ showed a greater difference in OS between PD-L1 positive and negative groups than those using $<50,{ }^{17,19,28}$ although no significant survival benefits were obtained in the former due to limited sample sizes. Moreover, the unfavorable prognostic value of PD-L1 was significantly seen in patients with esophageal cancer. ${ }^{16,17}$ Despite some trends observed, we currently cannot draw a valid conclusion that PD-L1 status is a predictor of prognosis for patients with gastric cancer or colorectal carcinoma. Considering the consistent trend of the prognostic significance of PD-L1 when using different kinds of anti-PD-L1 antibodies to stain tumor cells has been proven in another meta-analysis, ${ }^{30}$ subgroup analyses regarding source and type of antibody are not necessary.

\section{Limitations and future directions}

Efforts were made to conduct a comprehensive analysis to answer the question of expression rate of PD-L1 and the impact of PD-L1 status on patient prognosis in gastrointestinal tract cancer, but some limitations still should be acknowledged. First of all, we calculated or estimated 
A

\section{Study}

Ohigashi et al ${ }^{15}$

Hou et al ${ }^{20}$

Kim et al ${ }^{21}$

Qing et al ${ }^{23}$

Zhang et al ${ }^{25}$

Overall

$\mathbf{N}$

31

111

243

107

57

B

Study N

Chen et al ${ }^{17} \quad 99$

Geng et al ${ }^{19} \quad 100$

Liang et al ${ }^{28} \quad 185$

Zhao et $\mathrm{al}^{29} \quad 56$

Overall
Proportion: 95\% confidence interval

\section{Confidence interval}

$0.419(0.261,0.596)$

$0.631(0.537,0.715)$

$0.436(0.375,0.499)$

$0.505(0.411,0.598)$

$0.456(0.332,0.585)$

$0.495(0.415,0.576)$

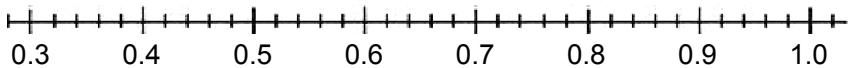

Proportion: $95 \%$ confidence interval
$\mathbf{N}$

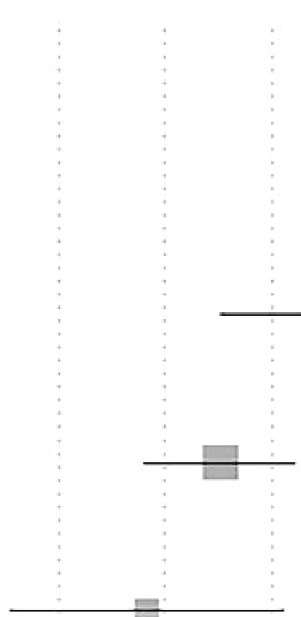

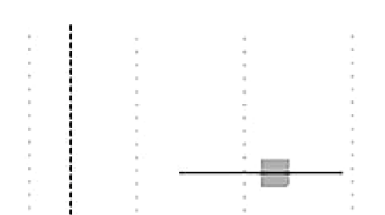

Confidence interval

$0.828(0.741,0.890)$

$0.650(0.552,0.737)$

$0.551(0.479,0.621)$

$0.482(0.355,0.611)$

$0.639(0.490,0.765)$

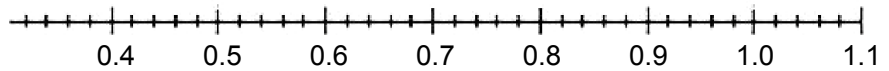

Figure 2 (Continued) 
C

\section{Study}

Loos et al $^{16}$

101

Jiang et $\mathrm{al}^{22} \quad 205$

Hua et $\mathrm{al}^{24} \quad 33$

Droeser et $\mathrm{al}^{26} \quad 1,420$

Shi et $a^{27} \quad 143$

Overall
Proportion: 95\% confidence interval
$\mathbf{N}$

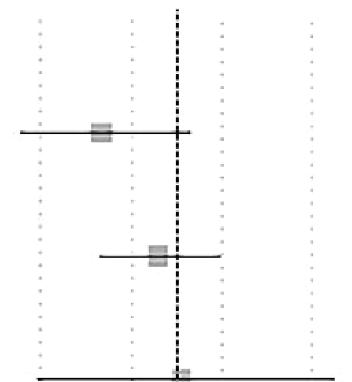

\section{Confidence interval}

$0.366(0.278,0.464)$

$0.429(0.363,0.498)$

$0.455(0.296,0.623)$

$0.471(0.445,0.497)$

$0.448(0.368,0.530)$

$0.449(0.417,0.483)$

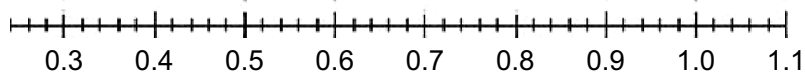

Figure 2 Single-arm meta-analysis of positive expression rate of programmed cell death I ligand I (PD-LI) on tumor cells in gastrointestinal tract cancer according to immunohistochemistry evaluation method and cut-off value.

Notes: (A) Percentage (cut-off value 10\%); (B) H-score (cut-off value $<50$ ); (C) $\mathrm{H}$-score (cut-off value $>50$ ).

\begin{tabular}{|c|c|c|c|}
\hline \multicolumn{2}{|c|}{ Overall survival } & \multirow{2}{*}{$\begin{array}{l}\text { HR (95\% CI) } \\
2.92(1.50,5.66)\end{array}$} & \multirow{2}{*}{$\begin{array}{l}\% \text { weight } \\
9.81\end{array}$} \\
\hline Loos et $\mathrm{al}^{16}$ & & & \\
\hline Chen et $\mathrm{al}^{17}$ & & $2.16(1.02,4.58)$ & 9.04 \\
\hline Wu et al ${ }^{18}$ & & $2.74(1.23,6.10)$ & 8.63 \\
\hline Geng et $\mathrm{al}^{19}$ & & $1.65(1.16,2.73)$ & 11.92 \\
\hline Kim et $\mathrm{al}^{21}$ & - & $0.65(0.42,1.02)$ & 11.76 \\
\hline Qing et $a^{23}$ & 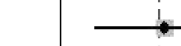 & $1.65(1.17,2.32)$ & 12.63 \\
\hline Droeser et $\mathrm{al}^{26}$ & + & $0.85(0.81,0.89)$ & 14.04 \\
\hline Shi et $\mathrm{al}^{27}$ & 1 & $2.61(1.01,3.58)$ & 10.09 \\
\hline Liang et $\mathrm{al}^{28}$ & & $1.74(1.20,2.71)$ & 12.07 \\
\hline Overall $\left(I^{2}=89.0 \%, P=0.000\right)(Z=2.46 \%, P=0.014)$ & & $1.61(1.10,2.35)$ & 100 \\
\hline${ }_{0.5}^{1}$ & $\begin{array}{l}1 \\
1.5\end{array}$ & & \\
\hline Favors PD-L1 positive & Favo & & \\
\hline
\end{tabular}

Figure 3 Total meta-analysis of programmed cell death I ligand I (PD-LI) positive versus PD-LI negative on tumor cells of gastrointestinal tract cancer patients in terms of overall survival.

Note: Weights are from random-effects analysis.

Abbreviations: $\mathrm{Cl}$, confidence interval; $\mathrm{HR}$, hazard ratio 


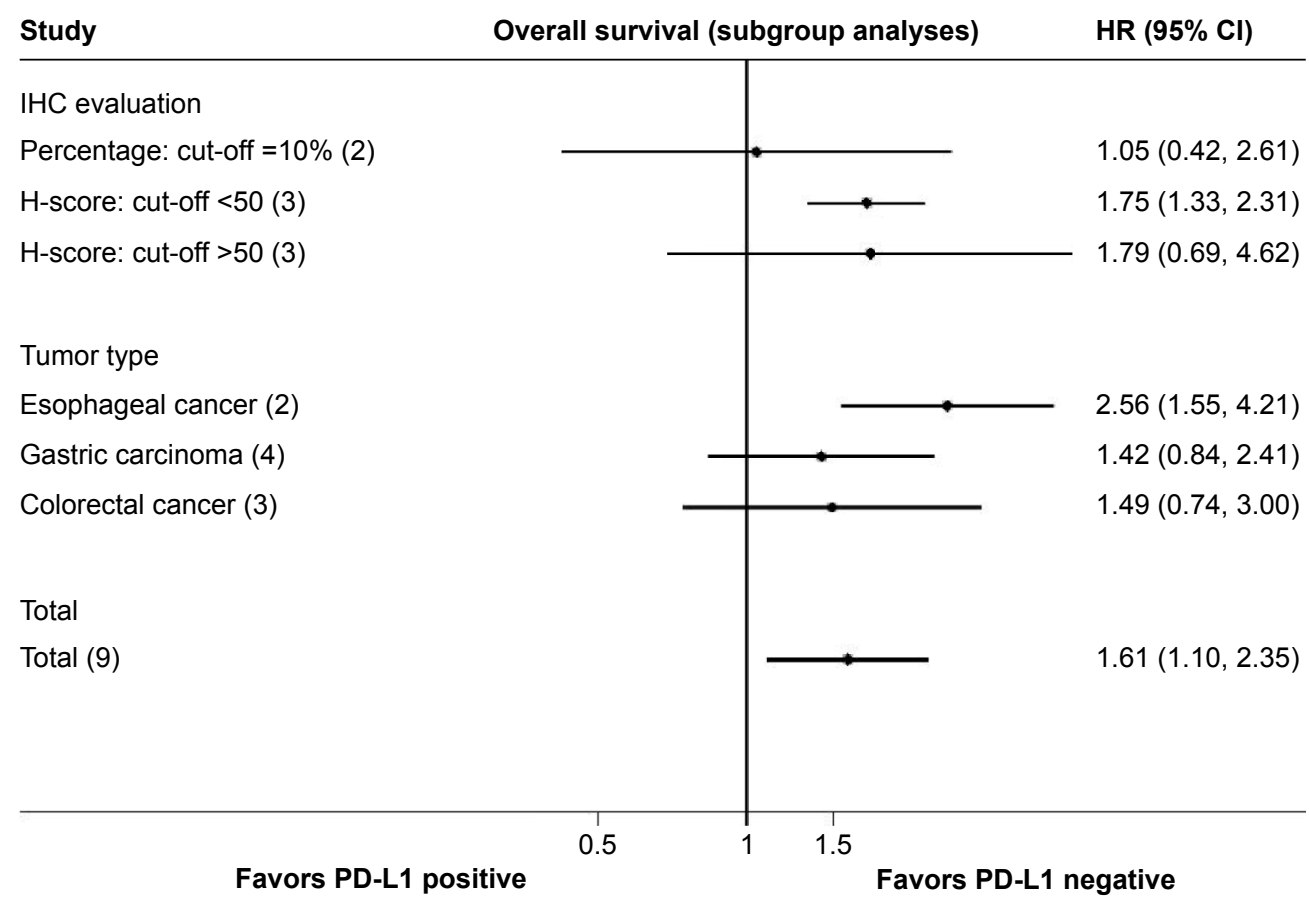

Figure 4 Subgroup analysis of programmed cell death I ligand I (PD-LI) positive versus PD-LI negative on tumor cells of gastrointestinal tract cancer patients in terms of overall survival.

Note: Weights are from random-effects analysis.

Abbreviations: $\mathrm{Cl}$, confidence interval; $\mathrm{HR}$, hazard ratio; IHC, immunohistochemistry.

some survival data of the HRs from the available data or Kaplan-Meier curves, which may have compromised the precision of the data. Secondly, cut-off values distinguishing positive and negative expression of PD-L1 determined by IHC evaluation and the primary antibodies varied in different types of tumors, which might cause heterogeneity of the overall results. The subgroup results should have addressed some concerns. Thirdly, we were not able to evaluate the expression rate of PD-L1 stratified by tumor type in subgroup analysis due to inconsistent IHC evaluations. Fourthly, we were unable to determine whether a correlation exists between positive PD-L1 expression and the clinical characteristics of gastrointestinal tract cancer. In addition, most of the eligible studies failed to provide data regarding progression-free survival or recurrence-free survival, so we only extracted OS data in our meta-analysis. Besides, scientists might prefer to only report the positive results of a prognostic biomarker, leading to the existence of potential publication bias. Further studies are warranted to address the above issues.

Regardless of the described limitations, this comprehensive analysis statistically confirmed almost half of all gastrointestinal tract cancer patients positively expressed PD-L1 in tumor cells and those PD-L1 positive patients were associated with significantly shorter OS, especially in esophageal cancer. A recent study has confirmed that positive PD-L1 expression is correlated with the improved efficacy of pembrolizumab, a drug marketed by Merck that targets the PD-1 receptor, in patients with advanced nonsmall-cell lung cancer. ${ }^{31}$ These results hint that, in clinical trials using anti-PD-L1 or anti-PD-1 antibodies as cancer immunotherapy, enrollment might also be preferentially carried out on patients with gastrointestinal tract cancer, especially esophageal cancer. Furthermore, more efforts should be made to investigate which cut-off value is the best for differentiating real PD-L1-positive patients with gastrointestinal tract cancer who would get survival benefit from PD-1/PD-L1 blockers.

\section{Conclusion}

In this meta-analysis, we found the positive expression rate of PD-L1 was nearly $50 \%$, no matter which method for IHC evaluation we chose. Additionally, we found positive PD-L1 expression status in tumor cells is a risk factor for prognosis in gastrointestinal tract cancer, especially in esophageal cancer.

\section{Acknowledgment}

This study was supported by the National Natural Science Foundation of China (number 81202069). 
The funder had no role in the study design, data collection and analysis, decision to publish, or preparation of this paper.

\section{Disclosure}

The authors declare no conflicts of interest in this work.

\section{References}

1. Siegel R, Naishadham D, Jemal A. Cancer statistics, 2012. CA Cancer J Clin. 2012;62(1):10-29.

2. Bjelakovic G, Nikolova D, Simonetti RG, Gluud C. Antioxidant supplements for preventing gastrointestinal cancers. Cochrane Database Syst Rev. 2008;(3):CD004183.

3. Yang S, Wu S, Huang Y, et al. Screening for oesophageal cancer. Cochrane Database Syst Rev. 2012;12:CD007883.

4. Markman JL, Shiao SL. Impact of the immune system and immunotherapy in colorectal cancer. J Gastrointest Oncol. 2015;6(2):208-223.

5. Ferrone S, Whiteside TL. Tumor microenvironment and immune escape. Surg Oncol Clin N Am. 2007;16(4):755-774.

6. Korman AJ, Peggs KS, Allison JP. Checkpoint blockade in cancer immunotherapy. Adv Immunol. 2006;90:297-339.

7. McDermott DF, Atkins MB. PD-1 as a potential target in cancer therapy. Cancer Med. 2013;2(5):662-673.

8. Chen J, Li G, Meng H, et al. Upregulation of B7-H1 expression is associated with macrophage infiltration in hepatocellular carcinomas. Cancer Immunol Immunother. 2012;61(1):101-108.

9. Wang A, Wang HY, Liu Y, et al. The prognostic value of PD-L1 expression for non-small cell lung cancer patients: a meta-analysis. Eur J Surg Oncol. 2015;41(4):450-456.

10. Zhou ZJ, Zhan P, Song Y. PD-L1 over-expression and survival in patients with non-small cell lung cancer: a meta-analysis. Transl Lung Cancer Res. 2015;4(2):203-208.

11. Pan ZK, Ye F, Wu X, An HX, Wu JX. Clinicopathological and prognostic significance of programmed cell death ligand1 (PD-L1) expression in patients with non-small cell lung cancer: a meta-analysis. $J$ Thorac Dis. 2015;7(3):462-470.

12. Tierney JF, Stewart LA, Ghersi D, Burdett S, Sydes MR. Practical methods for incorporating summary time-to-event data into meta-analysis. Trials. 2007;8:16.

13. Wells GA, Shea B, O'Connell D, et al. The Newcastle-Ottawa Scale (NOS) for assessing the quality if nonrandomized studies in metaanalyses [research tool]. Ottawa, ON: Ottawa Hospital Research Institute; 2014. Available at: http://www.ohri.ca/programs/clinical_ epidemiology/oxford.htm. Accessed August 1, 2015.

14. Begg CB, Mazumdar M. Operating characteristics of a rank correlation test for publication bias. Biometrics. 1994;50(4):1088-1101.

15. Ohigashi Y, Sho M, Yamada Y, et al. Clinical significance of programmed death-1 ligand-1 and programmed death-1 ligand-2 expression in human esophageal cancer. Clin Cancer Res. 2005;11(8):2947-2953.
16. Loos M, Langer R, Schuster T, et al. Clinical significance of the costimulatory molecule B7-H1 in Barrett carcinoma. Ann Thorac Surg. 2011;91(4):1025-1031.

17. Chen L, Deng H, Lu M, et al. B7-H1 expression associates with tumor invasion and predicts patient's survival in human esophageal cancer. Int J Clin Exp Pathol. 2014;7:6015-6023.

18. Wu C, Zhu Y, Jiang J, et al. Immunohistochemical localization of programmed death-1 ligand-1 (PD-L1) in gastric carcinoma and its clinical significance. Acta Histochem. 2006;108(1):19-24.

19. Geng Y, Wang H, Lu C, et al. Expression of costimulatory molecules B7-H1, B7-H4 and Foxp3+ Tregs in gastric cancer and its clinical significance. Int J Clin Oncol. 2014;20(2):273-281.

20. Hou J, Yu Z, Xiang R, et al. Correlation between infiltration of FOXP3+ regulatory $\mathrm{T}$ cells and expression of B7-H1 in the tumor tissues of gastric cancer. Exp Mol Pathol. 2014;96(3):284-291.

21. Kim JW, Nam KH, Ahn SH, et al. Prognostic implications of immunosuppressive protein expression in tumors as well as immune cell infiltration within the tumor microenvironment in gastric cancer. Gastric Cancer. 2014. Epub November 26.

22. Jiang D, Xu YY, Li F, Xu B, Zhang XG. The role of B7-H1 in gastric carcinoma: clinical significance and related mechanism. Med Oncol. 2014;31(11):268.

23. Qing Y, Li Q, Ren T, et al. Upregulation of PD-L1 and APE1 is associated with tumorigenesis and poor prognosis of gastric cancer. Drug Des Devel Ther. 2015;9:901-909.

24. Hua D, Sun J, Mao Y, Chen LJ, Wu YY, Zhang XG. B7-H1 expression is associated with expansion of regulatory $\mathrm{T}$ cells in colorectal carcinoma. World J Gastroenterol. 2012;18(9):971-978.

25. Zhang MY, Yang YY, Wang XH, Li XF. [Expression of Bcl-2, PD-L1 and its clinical significance in colorectal cancer.] Sichuan Da Xue Xue Bao Yi Xue Ban. 2012;43(6):827-829, 859. Chinese.

26. Droeser RA, Hirt C, Viehl CT, et al. Clinical impact of programmed cell death ligand 1 expression in colorectal cancer. Eur J Cancer. 2013; 49(9):2233-2242.

27. Shi SJ, Wang LJ, Wang GD, et al. B7-H1 expression is associated with poor prognosis in colorectal carcinoma and regulates the proliferation and invasion of HCT116 colorectal cancer cells. PLoS One. 2013;8(10):e76012.

28. Liang M, Li J, Wang D, et al. T-cell infiltration and expressions of T lymphocyte co-inhibitory B7-H1 and B7-H4 molecules among colorectal cancer patients in northeast China's Heilongjiang province. Tumor Biol. 2014;35(1):55-60.

29. Zhao LW, Li C, Zhang RL, et al. B7-H1 and B7-H4 expression in colorectal carcinoma: correlation with tumor FOXP3(+) regulatory T-cell infiltration. Acta Histochem. 2014;116(7):1163-1168.

30. Zhang Y, Kang S, Shen J, et al. Prognostic significance of programmed cell death 1 (PD-1) or PD-1 ligand 1 (PD-L1) Expression in epithelial-originated cancer: a meta-analysis. Medicine (Baltimore). 2015;94(6):e515.

31. Garon EB, Rizvi NA, Hui R, et al; KEYNOTE-001 Investigators. Pembrolizumab for the treatment of non-small-cell lung cancer. NEngl J Med. 2015;372(21):2018-2028. 


\section{Supplementary material}

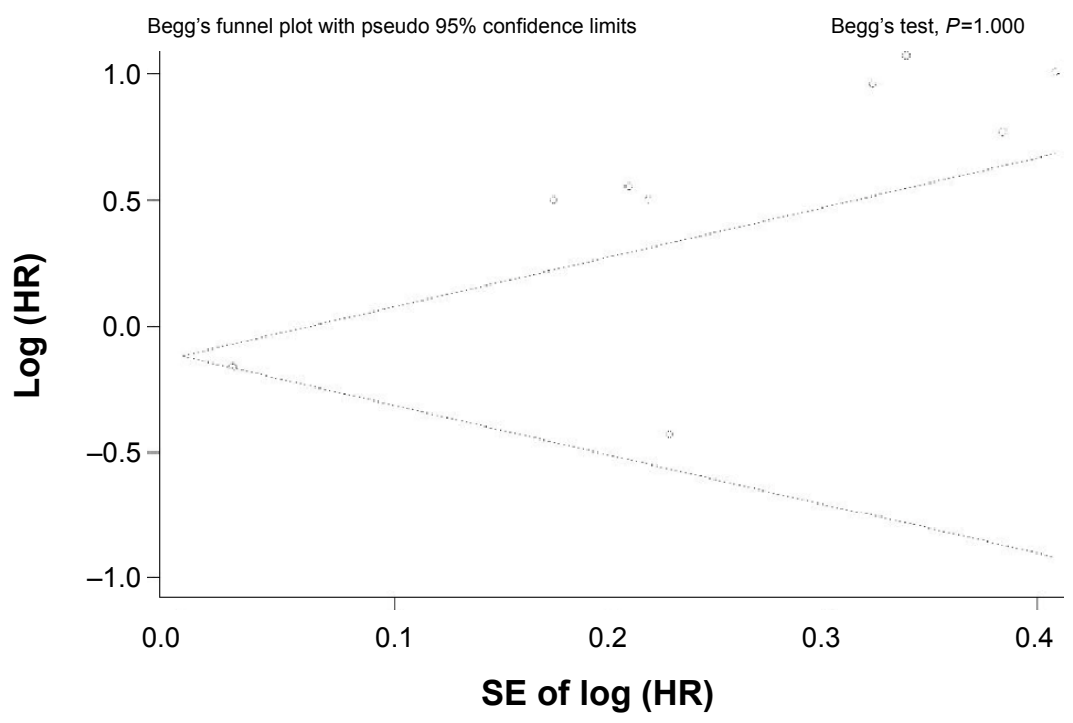

Figure SI Graphical funnel plots with Begg's test of the meta-analysis.

Abbreviations: $\mathrm{HR}$, hazard ratio; SE, standard error.

\section{Publish your work in this journal}

OncoTargets and Therapy is an international, peer-reviewed, open access journal focusing on the pathological basis of all cancers, potential targets for therapy and treatment protocols employed to improve the management of cancer patients. The journal also focuses on the impact of management programs and new therapeutic agents and protocols on patient perspectives such as quality of life, adherence and satisfaction. The manuscript management system is completely online and includes a very quick and fair peer-review system, which is all easy to use. Visit http://www.dovepress.com/testimonials.php to read real quotes from published authors.

\footnotetext{
Submit your manuscript here: http://www.dovepress.com/oncotargets-and-therapy-journal
} 